\title{
Return of sleep apnoea and sleep fragmentation following CPAP withdrawal in patients with obstructive sleep apnoea
}

\author{
To the Editor:
}

We previously published data in the European Respiratory Journal describing the return of obstructive sleep apnoea (OSA) following the withdrawal of continuous positive airway pressure (CPAP) [1]. In this study, about one third of patients did not experience a significant return of OSA by the fourth night without CPAP, as measured using the oxygen desaturation index (ODI). This suggested that CPAP need not be an every night therapy in some patients with OSA, although there was no clear way of identifying such patients in advance of the trial of CPAP withdrawal. Limitations to this study were discussed in the paper and correctly highlighted by VAN ZELLER and DRUMMOND [2]. In particular, it was possible that the ODI might not be sufficiently sensitive to sleep fragmentation occurring during the first nights of CPAP withdrawal. Although ODI derivatives and respiratory indices (with or without markers of arousal) have generally been found to be strongly correlated [3], and the ODI as good at predicting sleepiness as the apnoea-hypopnoea index (AHI) [4], it is possible that, in the early stages of OSA return, an arousal might tend to occur in advance of a significant desaturation event, perhaps due to a normalisation of the arousal threshold following a period on successful CPAP $[5,6]$. This would mean that using ODI values alone might underestimate the degree of sleep fragmentation and thus, potentially, the return of symptoms. This explanation has also been used to explain excessive daytime sleepiness occurring in the so-called upper airway resistance syndrome, in which there are respiratory events but oxygen desaturations are much smaller [7].

In order to explore this issue further we have looked at complete data from 184 patients having oximetry performed at home on each of the first four nights of CPAP withdrawal, from two centres (Oxford, $n=109$, and Zurich, $\mathrm{n}=75$ ) that were screening potential patients for inclusion in subsequent CPAP withdrawal trials. The criteria for study entry included an initial diagnosis of moderate-to-severe OSA, $>1$ year on CPAP, average CPAP use $>4 \mathrm{~h}$ per night, and an AHI (from machine download) on CPAP of $<10$. In the Oxford trials, the patients recruited had, on average, more severe OSA than the Zurich patients, which thus provided a wide range of OSA severity to study. We used the number of transient rises in heart rate of $>6$ beats per minute per hour of study as a surrogate of arousal frequency $[8,9]$, to explore if there was indeed evidence of a return of sleep fragmentation prior to the development of a significant ODI. This index is derived from a manually supervised automated analysis of the beat-to-beat heart rate (Visi-Lab; Stowood Scientific, Oxford, UK) which itself is derived from the oximeter's plethysmogram. Given the concerns over interscorer reliability when identifying EEG arousals [10], autonomic arousals could potentially be a more reliable marker of the increasing sleep fragmentation following CPAP withdrawal than EEG-defined events, and may also capture the more subtle events that presumably are less deleterious to sleep than those that incur full cortical arousal [11-13]. Heart rate responses to arousal have been shown to be reproducible within individuals, but there is considerable inter-individual variation in the relationship between EEG arousals and heart rate rises [14].

The mean \pm SD ODI $\left(>3 \%\right.$ ODI $\left._{3 \%}\right)$ on the fourth night of CPAP withdrawal was $28.8 \pm 19.8$, (Oxford $34.3 \pm 21.2$, Zurich 21.7 \pm 15.3 ). The mean heart rate arousal index (HRAI) on the fourth night was 34.4 \pm 20.4 , (Oxford 39.9 \pm 21.6 , Zurich 27.7 \pm 17.0 ). If the $\mathrm{ODI}_{3 \%}$ and HRAI on the first, second and third

@ERSpublications

Following CPAP withdrawal, in some obstructive sleep apnoea patients, evidence of sleep fragmentation can occur prior to the appearance of significant oxygen desaturations http://ow.ly/ OhoN30o957o

Cite this article as: Turnbull C, Pattenden S, Gaisl T, et al. Return of sleep apnoea and sleep fragmentation following CPAP withdrawal in patients with obstructive sleep apnoea. Eur Respir J 2019; 53: 1802351 [https://doi.org/10.1183/13993003.02351-2018]. 


\begin{tabular}{|c|c|c|c|c|}
\hline & Night 1 & Night 2 & Night 3 & Night 4 \\
\hline \multicolumn{5}{|c|}{ All patients following CPAP withdrawal } \\
\hline ODI >3\% & $26.0 \pm 17.4$ & $27.0 \pm 18.1$ & $28.6 \pm 19.1$ & $28.8 \pm 19.8$ \\
\hline $\mathrm{ODI}>3 \%$ as $\%$ of night 4 & $99 \pm 43$ & $106 \pm 53$ & $115 \pm 96$ & 100 \\
\hline HRAI & $34.4 \pm 20.4$ & $33.4 \pm 19.0$ & $34.2 \pm 19.2$ & $34.2 \pm 20.1$ \\
\hline HRAl as $\%$ of night 4 & $108 \pm 45$ & $108 \pm 52$ & $108 \pm 54$ & 100 \\
\hline \multicolumn{5}{|c|}{$\begin{array}{l}\text { Patients with gradual return of oxygen desaturations } \\
\text { following CPAP withdrawal }\end{array}$} \\
\hline ODI >3\% & $17.5 \pm 13.3$ & $25.8 \pm 18.9$ & $28.3 \pm 20.4$ & $33.9 \pm 20.6$ \\
\hline $\mathrm{ODI}>3 \%$ as $\%$ of night 4 & $51 \pm 14$ & $83 \pm 42$ & $83 \pm 22$ & 100 \\
\hline HRAl & $28.8 \pm 16.9$ & $30.8 \pm 16.8$ & $32.2 \pm 17.5$ & $34.9 \pm 16.8$ \\
\hline HRAl as $\%$ of night 4 & $85 \pm 43$ & $92 \pm 39$ & $94 \pm 35$ & 100 \\
\hline
\end{tabular}

Data are presented as mean $\pm S D$, for all patients $(n=184)$, and for those in whom there was a gradual return of obstructive sleep apnoea ( $n=37$ ), defined as an oxygen desaturation index (ODI) on the first night of CPAP withdrawal that was less than $70 \%$ of the ODI on the fourth night of CPAP withdrawal. HRAI: heart rate arousal index, number of rises in heart rate of more than 6 beats per minute.

nights of CPAP withdrawal are quoted as a percentage of the 4 th night value, then the return was $99 \pm 43 \%$, $106 \pm 53 \%, 115 \pm 96 \%$, and $108 \pm 45 \%, 108 \pm 52 \%, 108 \pm 54 \%$, respectively. Thus, from the overall data, on the first night of withdrawal, the $\mathrm{ODI}_{3 \%}$ and HRAI were already as high as that of night four (table 1).

In order to explore this further, we analysed the subgroup from both centres $(n=37)$ who only experienced a less than $70 \%$ return of their $\mathrm{ODI}_{3 \%}$ on night one of $\mathrm{CPAP}$ withdrawal compared to night four; individuals in whom the $\mathrm{ODI}_{3 \%}$ alone might have particularly underestimated the return of OSA. In this subgroup, by night four of CPAP withdrawal, there was no difference in severity compared to the whole group $\left(\mathrm{ODI}_{3 \%} 33.9 \pm 20.6\right.$ and $28.8 \pm 19.8$, HRAI $34.9 \pm 16.8$ and $34.2 \pm 20.1$ respectively). In these 37 subjects, although the $\mathrm{ODI}_{3 \%}$ was only $51 \pm 14 \%$ on the first night of CPAP withdrawal, compared to that of night four, the HRAI was already $85 \pm 43 \%$ that of night four (table 1). ANOVA with trend analysis showed no statistically significant trend across the four nights for HRAI $(\mathrm{p}=0.12)$, but there was a highly significant trend across the four nights for the ODI $(\mathrm{p}<0.001)$.

Whilst the majority of patients following CPAP withdrawal show a rapid rise in their ODI, we have shown that there are a minority of individuals who, despite a slower return of OSA as evidenced by the $\mathrm{ODI}_{3 \%}$, do in fact have evidence of a faster return of sleep fragmentation when this is measured by an autonomic marker of arousal. Thus the $\mathrm{ODI}_{3 \%}$ should perhaps not be used alone to assess whether patients can safely stop CPAP for short periods of time; an additional assessment of an arousal index, such as the HRAI, could perhaps be usefully included. These data would also support the idea that OSA and its associated sleep fragmentation blunt the arousal threshold, leading to longer respiratory events and thus larger hypoxic dips [6]. However, it should be noted that because autonomic markers of arousal, such as heart rate rises, are more sensitive to disturbing influences (for example they do not habituate to repetitive stimuli, as do EEG arousals [15]), it is not clear whether they are as important in leading to daytime symptoms, particularly if not propagated to the cortex [12]. Conversely, because there are some individuals in whom heart rate rises during an arousal are less pronounced, this may lead to an underestimation of the degree of sleep fragmentation in this situation. Just as there is a clear disconnect between respiratory events and the degree of EEG arousal [13], there can also be a disconnect between autonomic arousals and respiratory events characterised from oximetric indices.

Chris Turnbull ${ }^{1}$, Saxon Pattenden ${ }^{1}$, Thomas Gaisl $\oplus^{2}$, Valentina Rossi ${ }^{2}$, Sira Thiel $^{2}$, Malcolm Kohler ${ }^{2}$ and John Stradling ${ }^{1}$

${ }^{1}$ Oxford Centre for Respiratory Medicine and NIHR Biomedical Research Centre, Oxford, UK. ${ }^{2}$ Sleep Disorders Centre and Pulmonary Division, University of Zurich, Zurich, Switzerland.

Correspondence: John Stradling, Oxford Centre for Respiratory Medicine, Churchill Hospital, Oxford, OX3 7LJ, UK. E-mail: john.stradling@ouh.nhs.uk

Received: Dec 122018 | Accepted after revision: Feb 012019

Conflict of interest: C. Turnbull reports personal fees for consultancy from Bayer, outside the submitted work. S. Pattenden has nothing to disclose. T. Gaisl reports personal fees from Bayer AG (consultancy), outside the submitted 
work. V. Rossi has nothing to disclose. S. Thiel has nothing to disclose. M. Kohler reports grants from Swiss National Science Foundation, during the conduct of the study; grants and personal fees from Bayer, outside the submitted work. J. Stradling reports grants from Oxford Hospitals Charitable Funds, during the conduct of the study; grants and personal fees from Bayer, personal fees from ResMed UK, outside the submitted work.

Support statement: This work was supported by the Oxford Hospitals Charitable Funds and Schweizerischer Nationalfonds zur Förderung der Wissenschaftlichen Forschung. Funding information for this article has been deposited with the Crossref Funder Registry.

\section{References}

1 Rossi VA, Schwarz EI, Bloch KE, et al. Is continuous positive airway pressure necessarily an everyday therapy in patients with obstructive sleep apnoea? Eur Respir J 2014; 43: 1387-1393.

2 van Zeller M, Drummond M. CPAP holiday: are we there yet? Eur Respir J 2015; 45: 574-575.

3 Vazquez JC, Tsai WH, Flemons WW, et al. Automated analysis of digital oximetry in the diagnosis of obstructive sleep apnoea. Thorax 2000; 55: 302-307.

4 Bennett LS, Langford BA, Stradling JR, et al. Sleep fragmentation indices as predictors of daytime sleepiness and nCPAP response in obstructive sleep apnea. Am J Respir Crit Care Med 1998; 158: 778-786.

5 Haba-Rubio J, Sforza E, Weiss T, et al. Effect of CPAP treatment on inspiratory arousal threshold during NREM sleep in OSAS. Sleep Breath 2005; 9: 12-19.

6 Loewen A, Ostrowski M, Laprairie J, et al. Determinants of ventilatory instability in obstructive sleep apnea: inherent or acquired? Sleep 2009; 32: 1355-1365.

$7 \quad$ Pepin JL, Guillot M, Tamisier R, et al. The upper airway resistance syndrome. Respiration 2012; 83: 559-566.

8 Pitson D, Chhina N, Knijn S, et al. Changes in pulse transit time and pulse rate as markers of arousal from sleep in normal subjects. ClinSci 1994; 87: 269-273.

9 Johnson LC, Lubin A. The orienting reflex during waking and sleeping. Electroencephalog Clin Neurophysiol 1967; 22: 11-21.

10 Loredo JS, Clausen JL, Ancoli-Israel S, et al. Night-to-night arousal variability and interscorer reliability of arousal measurements. Sleep 1999; 22: 916-920.

11 Azarbarzin A, Ostrowski M, Hanly P, et al. Relationship between arousal intensity and heart rate response to arousal. Sleep 2014; 37: 645-653.

12 Martin SE, Wraith PK, Deary IJ, et al. The effect of nonvisible sleep fragmentation on daytime function. Am J Respir Crit Care Med 1997; 155: 1596-1601.

13 Jordan AS, Eckert DJ, Wellman A, et al. Termination of respiratory events with and without cortical arousal in obstructive sleep apnea. Am J Respir Crit Care Med 2011; 184: 1183-1191.

14 Azarbarzin A, Ostrowski M, Younes $\mathrm{M}$, et al. Arousal responses during overnight polysomnography and their reproducibility in healthy young adults. Sleep 2015; 38: 1313-1321.

15 Firth HW. Habituation of the orienting reflex during sleep. Electroencephalogr Clin Neurophysiol 1970; 28: 211. 